\title{
DRIH-MAC: A Distributed Receiver-Initiated Harvesting-aware MAC for NanoNetworks
}

\author{
Shahram Mohrehkesh, IEEE Student Member, Michele C. Weigle, IEEE Member and Sajal K. Das, IEEE Fellow
}

\begin{abstract}
In this paper, we introduce DRIH-MAC, a distributed receiver-initiated medium access control protocol for communication among nanonodes in a wireless electromagnetic nanonetwork. DRIH-MAC is developed based on the following principles: 1) communication starts via the receiver with the goal of maximizing the energy utilization; 2) the distributed scheme for accessing the medium is designed based on graph coloring; and 3) communication scheduling works in coordination with the energy harvesting process. DRIH-MAC is based on a probabilistic scheme to create a scalable and light-weight solution, which minimizes collisions and maximizes the utilization of harvested energy, and can be used in a wide variety of applications. Through simulation experiments, we demonstrate the efficiency of DRIH-MAC in a sample medical monitoring application. In particular, DRIH-MAC can improve energy utilization by $50 \%$ as compared to a random MAC protocol. Furthermore, it can satisfy application requirements such as delay, even with low energy harvesting rates.
\end{abstract}

Keywords-Nanonetworks, terahertz, energy harvesting, receiverinitiated, MAC protocol, energy harvesting-aware.

\section{INTRODUCTION}

The advancement of nanotechnology promises a new era of nano scale devices with computation and communication capabilities [2], [3]. These nanodevices can form a network of nanonodes, where each nanonode is composed of several nanomachines (e.g., nano-antenna, nano-memory, nanoprocessor, nanosensors, and energy storage) as illustrated in Figure 1. Each nanonode is in the range of nano to micro meters in size. A wide variety of emerging applications are envisioned for nanonetworks in the biological, medical, chemical, environmental, military, and industrial domains [3].

The functionalities of nanonodes are realized only through communication. Nanosensors will collect useful information such as the presence of bacteria or molecules, which must be sent outside of their sensing environment for storage and additional processing. In other words, they will need to communicate between themselves as well as with nodes in micro and macro domain networks, such as gateways connected to a local area network. Among all possible communication methods (e.g., molecular, optical, or acoustic communications) for nanonodes, studies [2] show that electromagnetic communication in the 0.110.0 terahertz $(\mathrm{THz})$ frequency band is a promising approach.

The tiny nanonodes will have a very limited energy storage capacity, probably in the form of ultra-nanocapacitors, which

\footnotetext{
Shahram Mohrehkesh and Michele C. Weigle are with the Department of Computer Science, Old Dominion University, Norfolk, Virginia, e-mail: smohrehk@cs.odu.edu, mweigle@cs.odu.edu.

Sajal K. Das is with the Department of Computer Science, Missouri University of Science and Technology, Rolla, Missouri, email: sdas@mst.edu.

Shahram Mohrehkesh is the corresponding author.

A preliminary version of this paper was presented at the ACM International Conference on Nanoscale Computing and Communication, Atlanta, GA, May 2014 [1].
}

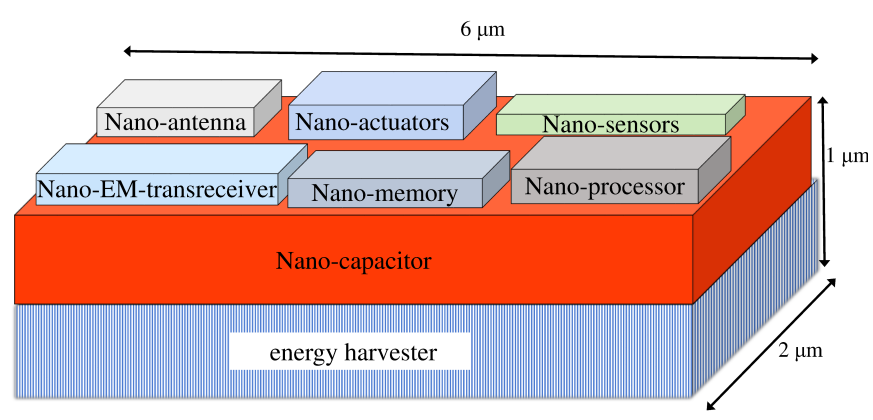

Fig. 1: Structure of a Nanonode - adapted from [3]

can only store enough energy for exchanging several hundred bits at a time [4]. Thus, a nanonode must harvest and store energy from ambient sources such as light, vibration, and biofuel. Nanoscale harvesting elements such as nanowires [5], which enable harvesting from vibration, will provide the possibility of communication for nanonodes. The variability of the energy sources makes the protocol design quite challenging. For example, in the medium access control (MAC) layer, coordination between nanonodes is required to make sure that a nanonode receiver will have enough energy to receive packets from a nanonode transmitter during communication. The design of energy harvesting-aware protocols that aim to maximize the utilization of available energy differs from traditional energyaware protocols that aim to minimize the consumption of energy. In nanonetworks, the energy is renewed, but the amount of available energy at any instance is limited. Hence, tailored energy harvesting-aware protocols for nanonetworks are required.

Designing protocols for accessing the medium is difficult due not only to energy availability, but also to special properties of nanonodes and nanonetworks. First, in most applications, coordination is required among hundreds of nanonodes which are also limited in their processing capabilities, implying that complex protocols cannot be considered. Moreover, traditional MAC mechanisms such as message exchange or handshake for synchronization prior to data transfer should be minimized in order to reduce the energy consumption as well as to enable the scalability of any solution. Finally, most energy harvesting-aware MAC protocols [6], [7], [8] in other networks are developed with two assumptions: 1) unlimited or very large energy storage; 2) very similar energy harvesting and consumption rates. However, these assumptions are not valid for limited size nanonodes [9]. As illustrated in Table I], when the nanonetworks are compared with mircoscale networks such as sensor networks, the magnitude of reduction in energy storage, energy consumption (for communication) and energy harvesting rates differs from the node size reduction. There- 
fore, these parameters should be studied differently in energy harvesting-aware design. Also, note that the energy storage and energy harvesting reduction is much higher than the energy consumption reduction, which result in dissimilar consumption and harvesting rates. Furthermore, these aspects become more critical in nanonetworks, where the data rates can be up to Tbps [10], [11]. Due to these challenges, novel MAC protocols for nanonodes are in great demand [12], [13]. This motivates our work in this paper.

In a preliminary work [1], we developed a receiver-initiated energy harvesting-aware MAC protocol (RIH-MAC), which is scalable and lightweight. Unlike traditional MAC protocols, which mainly focus on minimizing collisions and improving bandwidth efficiency, our solution relies on a receiverinitiated transmission for energy-harvesting nanonetworks. By coordinating the communication through the receiver in RIHMAC, a transmitter adaptively selects its participation in the network load, thus allowing RIH-MAC to achieve a low collision probability, a high packet delivery ratio, and high energy utilization. In fact, a transmission occurs only if there is a high probability that the receiver will have enough energy for the reception. RIH-MAC can operate in both centralized and distributed topologies of nanonetworks. The centralized solution [1] deals with topologies in which nanonodes are in direct communication with a more powerful device, called a nanocontroller [13], [15], which is responsible for scheduling communication with the nanonodes. In centralized RIH-MAC, packet scheduling is developed based on a probabilistic method while in the distributed solution, a distributed and predictive scheduling based on graph coloring is developed. The centralized solution is simpler to implement and has less overhead in terms of scheduling and energy consumption. However, in some applications of nanonetworks, it is not possible to provide direct communication between all nanonodes and the nanocontroller. In these situations, the distributed solution comes into play.

In this paper, we focus on the design and evaluation of a distributed implementation of the RIH-MAC protocol, called DRIH-MAC, which is suited for an ad hoc formation of nanonodes without a central point for scheduling communication. Each nanonode can directly communicate with other nanonodes in the neighborhood, and these neighbors provide connections to other nanonodes in the network. Our novel contributions in DRIH-MAC are: (i) a probabilistic and distributed coordinated MAC protocol that employs receiver-initiated transmissions, distributed edge-coloring, and predictive energy estimation, in order to control medium access in a scalable and harvestingaware fashion; (ii) a MAC protocol that outperforms a random MAC protocol in maximizing energy utilization by $50 \%$, thus resulting in better network performance; and (iii) a mac protocol that is applicable to a wide variety of nanonetwork applications. To demonstrate the characteristics of DRIH-MAC, we evaluate its performance in a sample medical monitoring application. Simulation experiments demonstrate that DRIH-MAC can help satisfy the application requirements such as delay, even with low energy harvesting rates.

The remainder of this paper is organized as follows. Section II introduces the system model of nanonodes and characterizes the nanonetwork in which DRIH-MAC will be used. Section III describes the DRIH-MAC protocol in detail, while Section IV] evaluates the distributed protocol through simulation experiments. Section $\mathrm{V}$ evaluates the performance of DRIHMAC in a sample medial monitoring application. Related work is presented in Section VI, while conclusions are offered in Section VII with directions for future research.

\section{System MODELS}

This section briefly introduces the underlying system models and assumptions as well as the application requirements for which DRIH-MAC is developed.

\section{A. Communication Model}

Nanonodes communicate in the $0.1-10 \mathrm{THz}$ frequency band [3], [4], which results in a micro to millimeter communication range [3], [15]. The nanonodes use pulse-based communication and Rate Division Time Spread On-Off Keying (RD TSOOK) [16] as the modulation mechanism, where a logical 1 is transmitted as a femto-second long pulse, and a logical 0 is transmitted as silence. To recognize symbols (silence or pulse), the inter-symbol time is set to several hundred multiples of the symbol duration. Due to the short duration of symbols, providing time synchronization could be a challenge. However, RD TS-OOK does not need tight synchronization. The time between symbols (pulses or silence) is fixed and is much longer than the pulse duration. This way, no tight synchronization among the nanosensor devices is required all the time. Only some nano-devices will be synchronized after detecting an initialization preamble and only for the duration of a packet transmission [16]. A receiver architecture has been proposed in [17] to detect symbols based on a continuous-time moving average. This simple receiver architecture provides a symbol detection scheme for implementation on nanonodes and also relaxes the synchronization requirements. We assume such a scheme exits that enables synchronization for communication among nanonodes.

\section{B. Energy Model}

Nanonodes need energy, mainly for their communication. Due to the limited size of nanonodes, they rely on energy harvesting methods, which nanoscale harvesters are required. We assume ambient vibration [3] as the main method for energy harvesting for nanonodes. The amount of energy harvested at each timeslot follows a stochastic process. Moreover, we consider an ultra nano-capacitor with non-linear behavior as the energy storage of each nanonode [4]. We assume that the energy storage is ideal and there is no significant leakage. We also consider that all nanonodes follow a similar harvesting model and that they have the same energy consumption model.

\section{Network Model}

We consider an ad hoc network of non-mobile nanonodes, each communicating with its neighbors. As illustrated in Figure 2. nanonode $\mathrm{A}$ has nanonodes $\mathrm{B}$ and $\mathrm{C}$ as its neighbors. A nanonode cannot transmit and receive at the same time. Each nanonode is responsible for forwarding the traffic of its neighbors until it reaches the nano-controller. We develop a model that provides the flexibility of increasing or decreasing the data traffic forwarding rate. The forwarding model has been inspired by approaches for solving the flooding (broadcast storm) problem in ad hoc networks [18], [19]. The policy for forwarding neighbors' traffic is guided by a probability of $\frac{g}{G}$, where $G$ is the number of neighbors and $g$ can take values from the real set of numbers in $\Re(0 . . G]$. Setting $g=G$ implies that the probability of packet forwarding is equal to 1 and results in flooding. 
TABLE I: Comparison of micro scale networks and nanonetwork

\begin{tabular}{c|lll} 
& Micronetwork & Nanonetwork & $\begin{array}{l}\text { Magnitude of Scale } \\
\text { Reduction }\end{array}$ \\
\hline Power Consumption & {$[14]$} & {$[4]$} & 3 \\
Node Size & $\mathrm{cm}^{3}$ & $\mu \mathrm{W}$ & 4 \\
Energy Storage & $\mathrm{J}$ & $\mu^{3}$ & 12 \\
Energy Harvesting Rate & $\mu \mathrm{J} / \mathrm{s}$ & $\mathrm{pJ}$ & 6
\end{tabular}

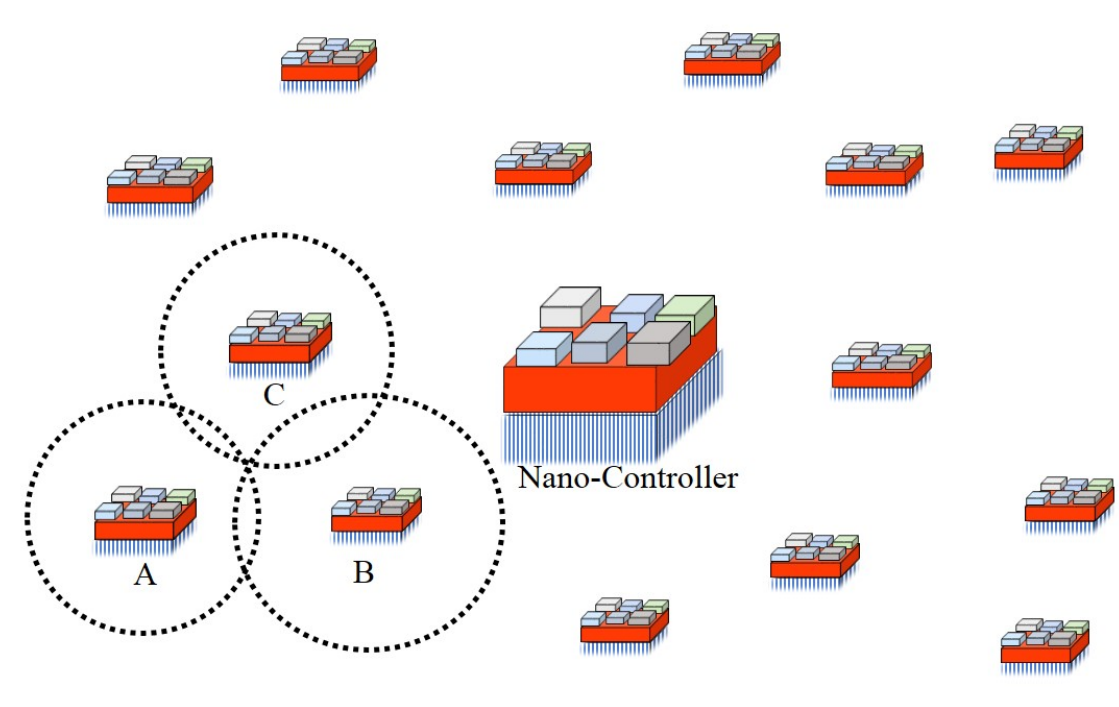

Fig. 2: Nanonetwork illustration - communication range is represented as dotted circles

\section{Application Requirements}

We assume that the applications for nanonodes can tolerate delays on the order of seconds. This assumption particularly applies to scenarios where the energy harvesting rate is lower than the consumption rate. In the $\mathrm{THz}$ band, the available bandwidth is very large (e.g., hundreds of gigabits per second). Therefore, the delay in packet transmission and propagation is on the order of picoseconds. The major delay imposed is from the time required to harvest enough energy to exchange packets.

Furthermore, since applications are not loss-sensitive, we consider only a simple acknowledgement scheme and a limited number of retries for unsuccessful transmissions. This will be the main mechanism to compensate for packet loss due to molecular absorption. It also handles any loss due to collisions of packets. We reduce the probability of collisions as part of our MAC design as discussed in Section III. Moreover, we are assuming that the packets are generated at a constant rate.

\section{RECEIVER-INITIATED ENERGY HARVESTING-AWARE MAC}

Our communication model between nanonodes is receiverinitiated, and the time is divided into equal timeslots. In each timeslot, up to two packets could be exchanged. The receiver announces that it is ready to receive a packet by sending ready to receive (RTR) to one or more nanonodes. The recipient of the $R T R$ packet may transmit a DATA packet accordingly. If required, the receiver can set a corresponding $A C K$ field in the next $R T R$ packet.

Figure 3 illustrates a sample sequence of RTR and DATA packets between a receiver (denoted as A) and a sender (de- noted as B), with a possible corresponding scenario of energy consumption and harvesting. When the first $R T R$ is transmitted by $\mathrm{A}, \mathrm{B}$ does not receive it, which could be due to several reasons such as lack of energy or it is already communicating with another node. In the illustrated scenario the energy level of $\mathrm{B}$ is $0 \mathrm{pJ}$, so it cannot receive the RTR packet. During this slot, the two nodes harvest $0.05 \mathrm{pJ}$ of energy. At the beginning of the next slot, the energy level of $A$ is $0.45 \mathrm{pJ}$ and that of $\mathrm{B}$ is $0.05 \mathrm{pJ}$. In the next slot, $\mathrm{B}$ receives the RTR packet, but does not transmit a DATA packet because of lack of energy (1 $\mathrm{pJ}$ is required). In the illustrated scenario, B does not transmit the DATA packet because it does not have enough energy, i.e., 1 pJ. During this slot, the two nanonodes harvest 2 pJ. Upon receiving the third RTR, B transmits a DATA packet and A receives it. This numerical example illustrates why coordination and scheduling of packet transmissions are required to avoid unsuccessful packet exchanges and therefore maximize the utilization of energy. The details of scheduling when to transmit and receive $R T R$ s is part of RIH-MAC to be described in this section. The $R T R$ packet, as illustrated in Figure 4, contains the node ID, destination ID (0 for broadcast), number of neighbors, maximum known degree, current amount of energy, mode of communication (centralized or distributed), and other fields that will be described in the reminder of this section.

There are two reasons for choosing the receiver-initiated communication model. First, in a centralized topology, the nanocontroller is responsible for the management of communication among nanonodes. Due to the higher energy budget of the nanocontroller and the need for more efficient usage of 


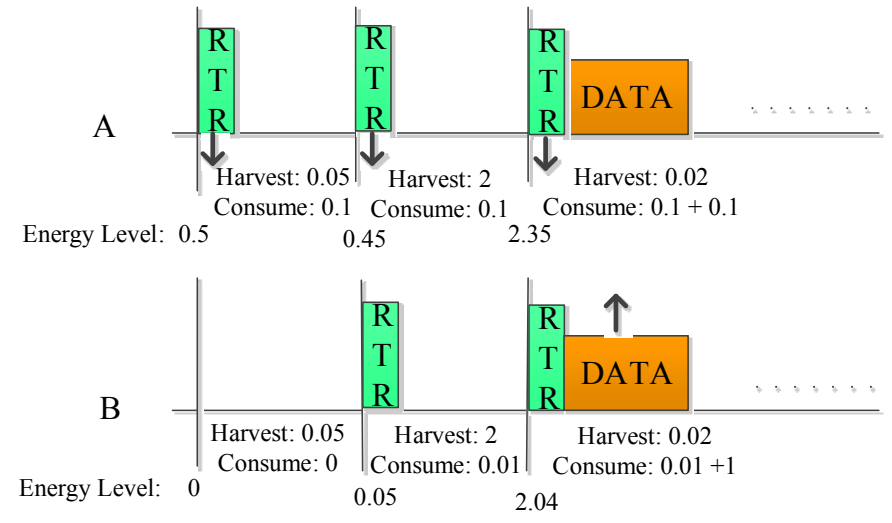

Fig. 3: RTR and DATA Packet Exchange - energy values in $\mathrm{pJ}$ based on energy harvester model in [4] and consumption model in [13], [20], [1]

\begin{tabular}{|c|c|}
\hline & 8 bits \\
\hline $1-2$ & Packet Sequence ID \\
$3-4$ & Node ID \\
\hline $5-6$ & Destination ID (0 reserved for broadcast) \\
7 & Number of Neighbors \\
8 & Maximum Known Degree \\
\hline $9-10$ & Current Amount of Energy \\
\hline 11 & Mode of Comm. \\
\hline $12-14$ & Ack (optional) \\
\hline 15 & Link Color \\
\hline $16-17$ & Rotation Offset Numbers \\
$18-25$ & Payload \\
\hline
\end{tabular}

Fig. 4: RTR Packet

energy on the transmitter side, the receiver-initiated communication model moves the burden of energy consumption for the management of communication and packet handling to the nanocontroller. Furthermore, since it is assumed that there are abundant nanonodes, a significant fraction may not be able to transmit a packet at each time slot. So, the receiver-initiated method enables a fair traffic flow from different nanonodes without being concerned about the energy level of nanonodes.

Second, it is better to initiate communication only when it is most likely that the receiver will have enough energy to receive a packet. Otherwise, many transmissions would be unsuccessful because of a high possibility of the receiver not having enough energy. Note that handshaking may not be an efficient method for the small packet sizes that nanonodes can handle. However, there is still a need for scheduling, which is more complex for the distributed communication model. In the following, we will introduce our scheduling scheme for the distributed RIH-MAC protocol.

\section{A. Graph Model}

Our medium access method relies on the receiver-initiated principle and distributed scheduling for nanonodes, which is energy-efficient, energy-adaptable, lightweight, and scalable. Energy-adaptable means that the scheme is adaptable to various energy harvesting rates. Our scheme uses distributed scheduling for communication among nanonodes. Communication between a group of ad hoc formed nanonodes can be modeled as an edgecoloring problem, which is to determine the minimum number of colors needed to color the edges of a graph such that two edges incident on a common node do not have the same color. Each pair of nanonodes that are in the communication range of each other will have an edge between them. All incident edges of a node should have different colors. Each color represents the timeslot in which a nanonode can communicate with one of its neighbors.

The edge coloring problem is NP-complete, and by Vizing's theorem [21], the minimum number of colors needed to edge color a graph is either its maximum degree $\Delta$ or $\Delta+1$. Most edge coloring solutions are centralized. Here, we are looking for a lightweight distributed solution. Among distributed solutions, we adopt the proposed solution in [22] that runs in $O(\log \log n)$ rounds, where $n$ is the number of nodes in the graph. The method finds a coloring solution for the problem with a high probability close to 1 . Most of the other distributed and deterministic models such as [23] are more complex and also do not offer a significant performance improvement. However, this algorithm satisfies the simplicity and distributed properties that we require. When this scheme fails to color properly, it can be run again at a low cost. Note that even though a network of nanonodes will be mainly static, its formation and topology can be dynamic over time (due to failure of nanonodes, or adding or removing some nanonodes), and therefore coloring will need to be run again.

We color a graph with $(1+\epsilon) \Delta$ colors, for any positive $\epsilon<1$. Our distributed edge coloring algorithm is shown in Algorithm 11 Each edge $w=(u, v)$ between two arbitrary nanonodes $u$ and $v$ is initially given a palette of $(1+\epsilon) \Delta$ colors. This palette is recorded locally at each nanonode. The formation of this palette is also done through receiving and transmitting some initial RTR packets where no DATA packets are sent in reply. A new nanonode that has no color assigned for its edges will transmit zero in the color field of its RTR packet. The main coloring process occurs in rounds. In each round, each uncolored edge independently picks a tentative color uniformly at random from its current palette. If no other edges of nodes $u$ and $v$ are using this color, it is picked as the final color of edge $w$. Otherwise, the coloring of this edge will be tried again in the next round. At the end of each round, the palettes are updated in the obvious way: colors successfully assigned are deleted from the current palette. The duration of each round would be equal to the exchange of $R T R$ packets to announce the selected colors and receiving the selected colors from neighbors. Therefore, to reach the agreement or disagreement on a color with all neighbors through $R T R$ packets, at most $\Delta+1$ timeslots are required for each round with the assumption of no $R T R$ packet failure. More rounds are required for these circumstances. A colored graph is illustrated in Figure 5. Colors are also labeled with numbers. 

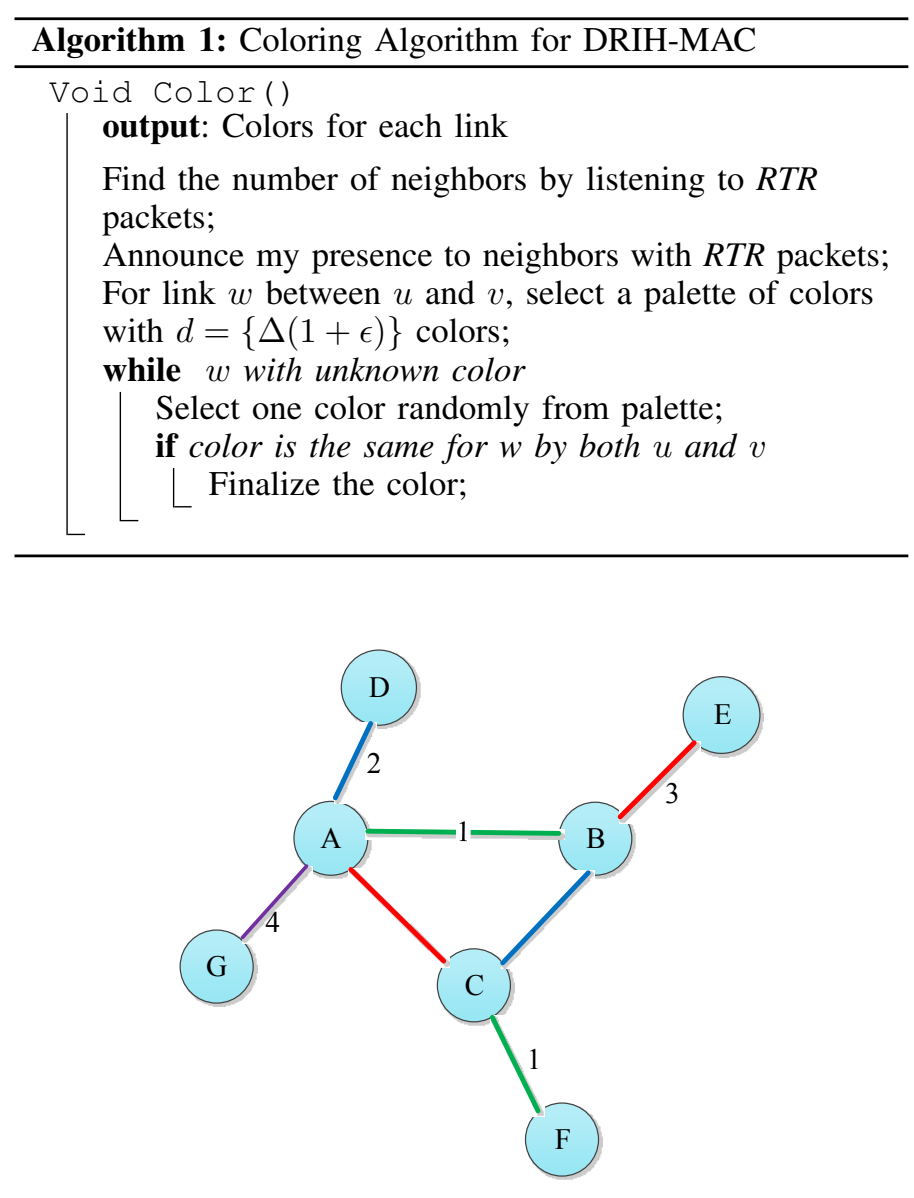

Fig. 5: A Colored Graph. Here each number represents a different color.

We consider the performance of edge coloring when several nanonodes with a communication range of $10 \mathrm{~mm}$ are distributed uniformly in a cube of size $100 \times 100 \times 10 \mathrm{~mm}$. Figure 6 shows the probability of successful coloring of the graph for various values of $\epsilon$. As can be seen for all values, the probability of success is more than $99 \%$, and the higher $\epsilon$, the higher the probability of successful coloring.

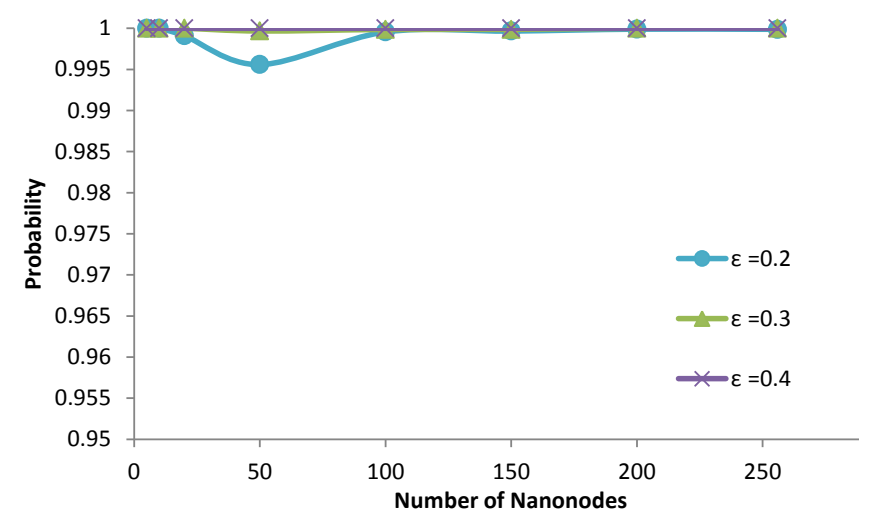

Fig. 6: Probability of Successful Edge Coloring

\section{B. An Illustrative Example}

Figure 7 depicts the number of rounds required until all edges are colored properly. Clearly, for a higher number of nanonodes, it takes more rounds to color, but it still is a reasonable number of rounds. Recall that the duration of one round is equal to the length of $2 \cdot(\Delta+1)$ timeslots. Since the duration of RTR packets is very short, the scheme converges quickly, e.g., less than one nanosecond in the scenario with no energy limit and 256 nanonodes.

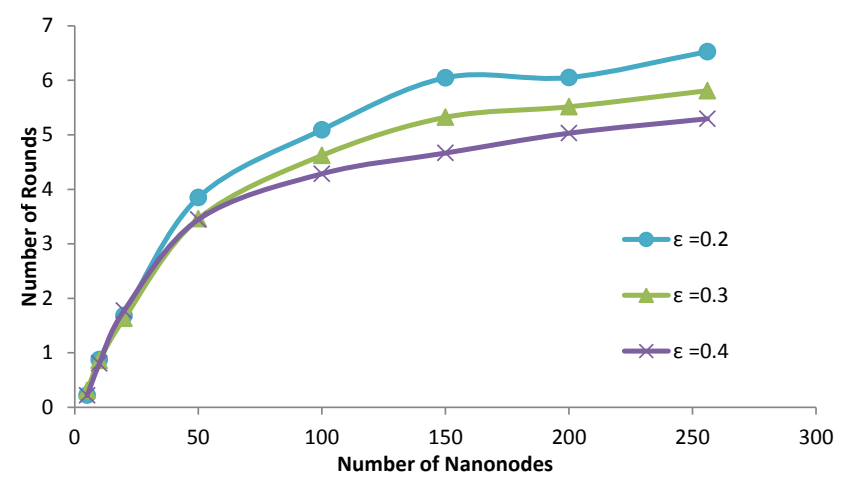

Fig. 7: Number of Rounds to Color Edges

After coloring, we need to specify the communication direction between two nanonodes on the link between them. One way to schedule the direction of communication is to extend the coloring algorithm to assign two colors per edge. However, since we assume a nanonode cannot transmit and receive at the same time, it would be similar to switching between the transmission and reception states, consecutively.

For simplicity, we assume consequent changes of the communication direction as shown in Figure 8. A node with a lower ID, here alphabetically ascending, sends in the first slot and receives in the following slot for each link. For example, for the link with color 2 between nodes $B$ and $C$, first $B$ plays the role of sender at slot 3 (depicted as $2 \mathrm{~S}$ ) and $C$ plays the role of receiver (depicted as $2 \mathrm{R}$ ). In the next slot (slot 4 ), $B$ receives (depicted as $2 \mathrm{R}$ ) and $C$ transmits (depicted as $2 \mathrm{~S}$ ). Recall that the exchange of RTR and DATA packets occurs in each timeslot with the receiver initiating. Note that slots 7 and 8 are not used by $B$ and $C$. It may appear to be a waste of slots, however, this is the cost for communication without collision. We call these eight timeslots a cycle, which is repeated over and over.

By exploiting the coloring mechanism, DRIH-MAC avoids collisions due to concurrent transmissions and is preferred over random access methods. First, the generated data rates of nanonodes are similar to each other, so there is no need to provide more access to the medium for one nanonode over another. Second, although there could be timeslots in DRIH-MAC which are not used by nodes with fewer neighbors, it is acceptable in scenarios where the energy harvesting rate is lower than the consumption rate, because some slots eventually will not be utilized due to lack of energy. An optimum energy consumption mechanism can coordinate its communication schedule with these empty slots to maximize energy and timeslot utilization. We describe this further in the next subsection. 


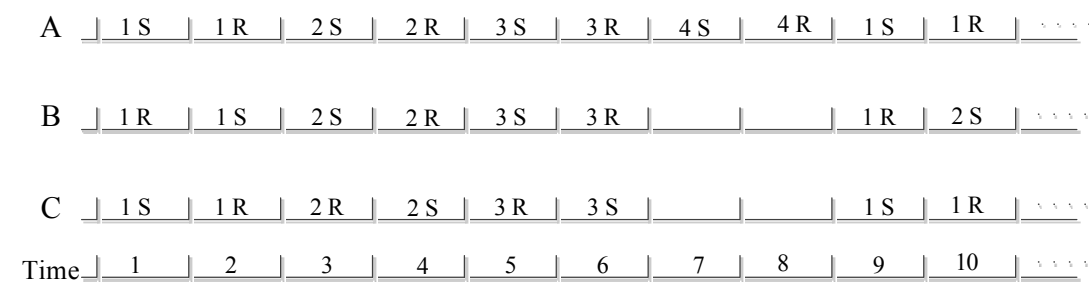

Fig. 8: Example Communication in DRIH-MAC. The nanonodes $A, B$ and $C$ from Figure 5 are shown. $\mathrm{S}$ indicates the sending mode, and $\mathrm{R}$ indicates receiving mode. The number preceding $\mathrm{S} / \mathrm{R}$ indicates the color.

\section{Coordinated Energy Consumption Schedule}

After the coloring of the graph is done, the proposed DRIHMAC protocol can be executed stand-alone if there is no energy limitation on nanonodes. However, a coordinated energy consumption schedule (CECS) between two communicating nanonodes is required to achieve the highest performance. When there is no such coordination, many $R T R$ packets would be sent with no DATA packet response. Similarly, transmitters may listen to receive $R T R$ packets but receive no $R T R$ packets due to lack of energy at the receiver side to transmit the RTR packet. In both scenarios, energy is wasted. Therefore, the CECS scheme tries to predict the energy level of each neighbor nanonode as well as their next consumption model to avoid these situations. Since the process of energy harvesting for neighbor nanonodes is not known exactly, CECS would be predictive. However, as we will show, the prediction developed in CECS still improves energy consumption significantly.

We assume that all nanonodes follow a similar harvesting model. The amount of available energy is received from each neighbor through $R T R$ packets, which also contain the number of neighbors. We assume nanonodes have a policy that specifies how much energy to spend per level of energy for each neighbor. An optimum policy can be solved by modeling the problem as a Markov decision process, and then the offline result can be stored as a lookup table [24], [9]. The different levels of available energy define the states of the stochastic process. Harvesting energy transits the node's state to a state with a higher level of available energy. Also, for each state, an optimum policy indicates the amount of energy to be used for communication with neighbors. In general, the optimum policy specifies higher energy consumption amounts for states with higher amount of available energy. Likewise, lower energy consumption is assigned for lower energy states.

Once there is an optimum energy consumption policy, we define the amount of energy consumption per cycle. For example, in Figure 5 for nanonode $B$ with 3 neighbors, if, at an arbitrary cycle $C_{i}$, the optimal policy determines that only two packets can be received from the neighbors, CECS indicates the policy 2 , in which links 1 and 2 are selected for communications. This selection is represented as a pattern of $0 \mathrm{~s}$ and $1 \mathrm{~s}$. Table IIa presents a sample pattern for node $B$. At cycle $C_{i}$, the selected policy for node $B$ would be policy number 2 as shown in Table IIb Next, at cycle $C_{i+1}$, based on the amount of energy, the oprimal policy selects another pattern, for example policy 1 in Table Ic

To provide a fair data flow among all neighbors, the selected pattern rotates at the end of each cycle. For example, the pattern

TABLE II: Patterns Corresponding to Various Policies for Node $B$ with 3 Links (policy number is equal to the number of receptions in one cycle)

\section{(a) Pattern}

\begin{tabular}{c|ccc} 
Policy & Pattern \\
\hline 0 & $\begin{array}{l}0 \\
0\end{array}$ & 0 \\
1 & 1 & 0 & 0 \\
2 & 1 & 1 & 0 \\
$\geq 3$ & 1 & 1 & 1
\end{tabular}

(b) Selected Pattern for Cycle $C_{i}$

\begin{tabular}{|c|c|}
\hline Policy & Pattern \\
\hline 0 & $\begin{array}{llll}0 & 0 & 0\end{array}$ \\
\hline 1 & 100 \\
\hline 2 & 110 \\
\hline$>3$ & 111 \\
\hline
\end{tabular}

(c) Shift in Pattern for Cycle $C_{i+1}$

\begin{tabular}{|c|c|}
\hline Policy & Pattern \\
\hline 0 & $\begin{array}{llll}0 & 0 & 0\end{array}$ \\
\hline 1 & 100 \\
\hline 2 & $\begin{array}{llll}0 & 1 & 1\end{array}$ \\
\hline$>3$ & 111 \\
\hline
\end{tabular}

for policy 2 after rotation would be 011 at cycle $C_{i+1}$, and remains until the next selection of policy 2, after which the pattern will rotate again. All nanonodes will use the same pattern for different levels of energy. Table III] shows the pattern for nodes with four and five links. Although nanonodes follow the same pattern, they will be independent in their own rotation. The rotation offset number for each nanonode is transferred in the RTR packets. Moreover, the patterns for transmission and reception are independent. A receiver decides to transmit its $R T R$ if it predicts that the transmitter is scheduled to receive the $R T R$ based on the previous received rotation offset number. However, since this prediction can be incorrect, some RTRs may still be wasted, and consequently no DATA reply is received. This is avoidable only if the nanonodes decide about their energy consumption optimization model together, which seems to be implementable only with methods having significant overhead such as periodic status update packet exchange. Therefore, here we do not evaluate such a solution.

TABLE III: Patterns Corresponding to Various Policies

(a) Pattern for 4 Links

\begin{tabular}{|c|c|}
\hline Policy & Pattern \\
\hline 0 & $\begin{array}{lllll}0 & 0 & 0 & 0\end{array}$ \\
\hline 1 & $\begin{array}{llll}10 & 0 & 0\end{array}$ \\
\hline 2 & $\begin{array}{lllll}0 & 1 & 0 & 1\end{array}$ \\
\hline 3 & $\begin{array}{llll}0 & 1 & 1 & 1\end{array}$ \\
\hline$\geq 4$ & 1111 \\
\hline
\end{tabular}

(b) Pattern for 5 Links

\begin{tabular}{|c|c|}
\hline Policy & Pattern \\
\hline 0 & $\begin{array}{llllll}0 & 0 & 0 & 0 & 0\end{array}$ \\
\hline 1 & $\begin{array}{llllll}1 & 0 & 0 & 0 & 0\end{array}$ \\
\hline 2 & $\begin{array}{llllll}0 & 1 & 0 & 1 & 0\end{array}$ \\
\hline 3 & $\begin{array}{lllll}10 & 1 & 0 & 1\end{array}$ \\
\hline 4 & $\begin{array}{llllll}0 & 1 & 1 & 1 & 1\end{array}$ \\
\hline$\geq 5$ & $\begin{array}{lllll}1 & 1 & 1 & 1 & 1\end{array}$ \\
\hline
\end{tabular}


At each timeslot of a cycle, the transmitter waits to receive a $R T R$ from the receiver, only if based on the pattern, it is expecting a $R T R$ from the receiver. Similarly, a receiver will transmit a $R T R$ only if based on the transmitter pattern, it predicts that the transmitter will be waiting for a $R T R$ to send its DATA. Note that these controls and predictions are simple enough to run on a nanonode. Through this method, the transmitter does not consume energy for the reception of $R T R$ when one is not sent. Also, the receiver will not transmit any $R T R$ if it predicts that the transmitter is not scheduled to receive the $R T R$ and send a DATA packet.

A detailed analysis that ensures the existence of slots in which both the transmitter and receiver will be scheduled to send and receive at the same time can be found in Appendix A. Briefly, it can be described as follows. When the transmitter and receiver do not happen to be in 1 of their pattern at the same timeslot, they will jump into other states of energy due to changes in energy consumption and harvesting. Therefore, they will go to another state and pattern where they will eventually exchange packets. To make it clearer, we also show the measurements in simulation results, which numerically analyze the performance of CECS.

\section{PERFormance EVAluation}

We ran several simulation experiments in ns-3 to evaluate the performance of DRIH-MAC. For our simulation, we modified and enhanced the Nanosim module [25], which enables simulation of electromagnetic nanonetworks in ns-3. The major modifications were applied to the energy module and channel model. Nanonodes have harvesters that follow the harvesting model developed in [4]. To evaluate the effect of harvesting rate, we characterize the harvesting rate as a probability distribution function, where it is discretized to adapt to the simulation environment. Each nanonode has an ultra-nanocapacitor as the energy storage with a capacity of 100 picojoules.

Nanonodes are considered to be operating in an environment with $10 \%$ water vapor with the corresponding channel path loss model [4] in the 100-300 GHz frequency band. Energy consumption is modeled as 1 femtojoule for the transmission of each pulse and 0.1 femtojoule for the reception of each pulse [4], [13], [20]. The size of packets is selected based on our previous work [26], where we model and find the optimum packet size for several optimization functions. In these experiments, we set the size of $R T R$ packets to 25 bytes and the size of DATA packets to 250 bytes. There is always a back-log of packets ready in a queue to transmit. In this scenario, nanonodes are distributed uniformly in a cube of size $100 \times 100 \times 10 \mathrm{~mm}$.

\section{A. Overhead of Distributed Coloring}

Coloring the graph has an overhead in terms of energy consumption and delay when compared with a centralized solution of RIH-MAC. As described in Section III, it will take several rounds for the graph to be colored. The number of rounds does not significantly change with an increase in the number of nanonodes. The required energy for this operation is about 6 pJ per node for a nanonetwork with 300 nanonodes. Although this energy is not significant, depending on the energy harvesting rate, it may take several seconds to harvest the required energy for this purpose. Figure 9 illustrates the delay in coloring a nanonetwork. As viewed, the delay could be more than 2 minutes when the energy harvesting rate of $1 \mathrm{pJ} / \mathrm{s}$ is considered for a network of 256 nanonodes.

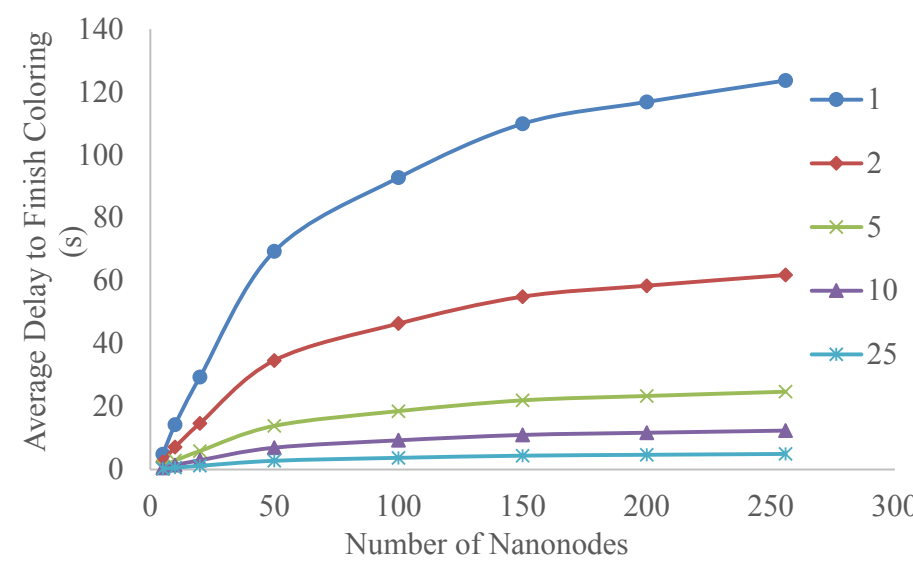

Fig. 9: Avergae Delay vs. Number of Nanonodes for Various Exponential Energy Harvesting Rates

\section{B. Coordinated Energy Consumption Schedule (CECS)}

To evaluate the performance of CECS, we define the following metric.

$$
R T R_{-} \text {Success }=\frac{R T R_{c}}{R T R_{c}+R T R_{u}+R T R_{w}},
$$

where $R T R_{c}$ is the number of RTRs with a successful DATA response, $R T R_{u}$ is the number of $R T R s$ which are not heard by the targeted sender due to lack of energy, and $R T R_{w}$ is the number of RTRs which are received, but cannot be replied to due to lack of energy. Note that for CECS $R T R_{w}=0$ since a nanonode will not listen to RTRs if it knows that it will not have energy for transmission.

Figure 10 illustrates the performance of CECS in comparison to the scenario where there is no scheduling of the transmission of RTRs. CECS achieves close to $100 \%$ success as the harvesting rate increases. The no-CECS case has a slower slope of improvement. The RTR success percentage is independent of the number of nanonodes as illustrated in Figure 11.

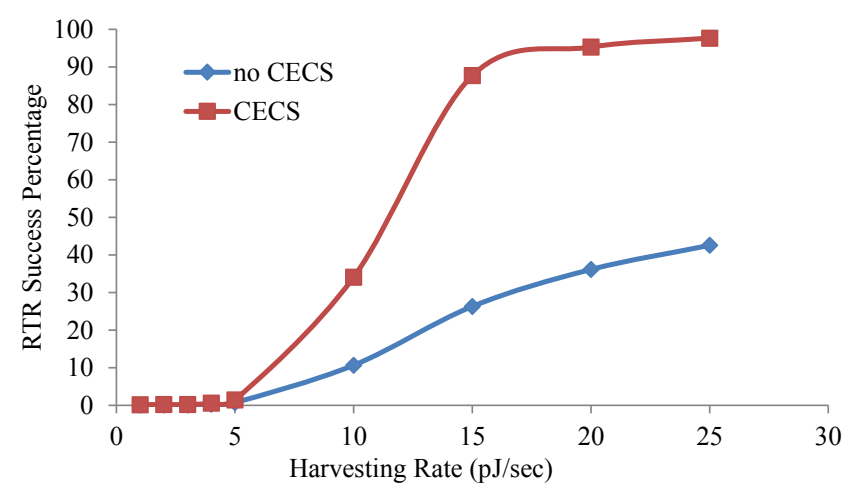

Fig. 10: RTR Success with Exponential Energy Harvesting Rate

In general, as the harvesting rate is increased, RTR_Success becomes closer to $100 \%$ because the energy would exist at all times, and $R T R_{u}$ becomes zero. This observation can also be seen in Figure 12, where the no-CECS scheme becomes closer to the CECS faster for the lognormal distribution of 


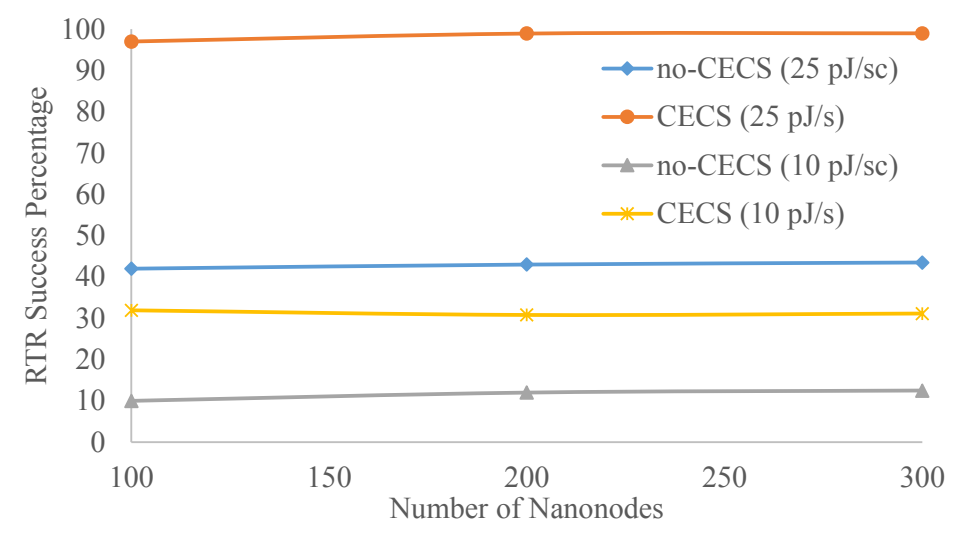

Fig. 11: RTR Success vs. Number of Nanonodes with Exponential Energy Harvesting Rate

energy arrival as compared to the exponential distribution used in Figure 10

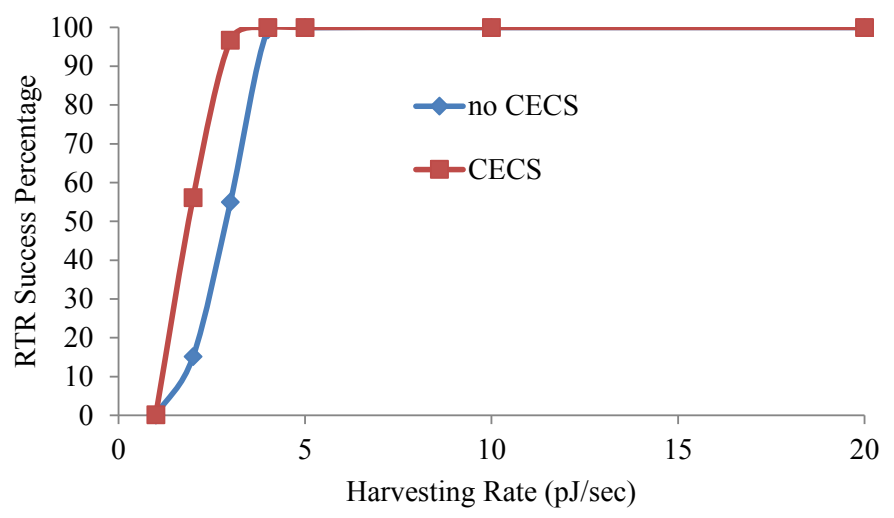

Fig. 12: RTR Success with Lognormal Energy Harvesting Rate, $\sigma^{2}=0.5 \cdot \mu$

Next, we measure the fairness index for communication with neighbors. Let $x_{i}$ represent the number of successful packet exchange with $i$ th neighbor, then the fairness index for communication with $n$ neighbors is defined as

$$
\mathcal{J}\left(x_{1}, x_{2}, \ldots, x_{n}\right)=\frac{\left(\sum_{i=1}^{n} x_{i}\right)^{2}}{n \cdot \sum_{i=1}^{n} x_{i}^{2}} .
$$

As shown in Figure 13, CECS achieves a better fairness index than the random selection of neighbors at each cycle. Furthermore, it can be observed that with an increase in the harvesting rate, fairness is increased, which actually occurs because of a more successful chance of message reception. The fairness index, indeed, confirms that not only will CECS result in communication between a nanonode and all of its neighbors, but it will do so in a balanced fashion.

\section{Capacity Utilization}

In this experiment, we want to evaluate the performance of DRIH-MAC with a random (receiver-initiated without CECS) MAC protocol to evaluate the utilization of energy with varying harvesting rates. In this scenario, a node will transmit RTR

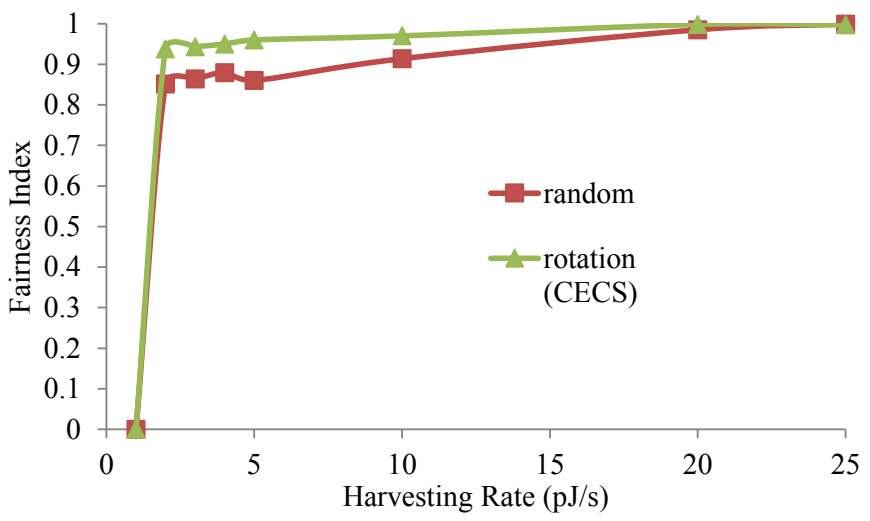

Fig. 13: Fairness Index vs. Harvesting Rate - Exponential Energy Harvesting Rate

packets constantly. That is, immediately after the reception of the corresponding DATA packet, it will transmit the next $R T R$ and so on. Clearly, with lack of energy for either the transmission or reception, the packet transfers will not occur. Energy is utilized properly only if a transmitted packet is received. Therefore, the utilization is defined as

$$
\mathbb{U}=\frac{R e c v}{R e c v_{\max }},
$$

where Recv represents the number of successful receptions and $R e c v_{\max }$ represents the number of receptions for the maximum harvesting rate.

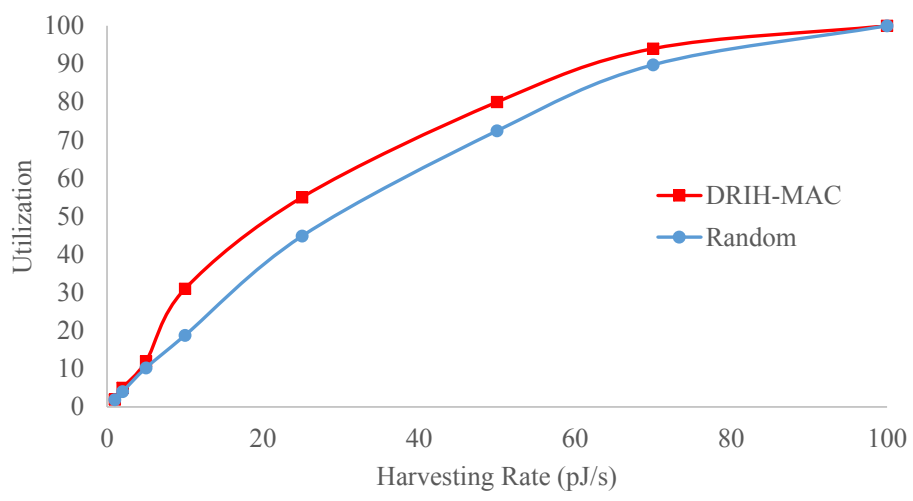

Fig. 14: Energy Utilization of a Single Link

As shown in Figure 14, DRIH-MAC outperforms a random transmission of packets. The difference is higher for a moderate harvesting rate, i.e., 5 to $50 \mathrm{pJ} / \mathrm{s}$. In this scenario, there are no collisions between these two nanonodes.

To investigate the effect of collisions where there is more than one neighbor, we evaluate a scenario where 100 nanonodes are distributed uniformly in a cube of $1 \times 1 \times 0.05 \mathrm{~cm}^{3}$. The utilization $\mathbb{U}$ is illustrated in Figure 15 DRIH-MAC again performs better than the random transmission of packets, with a utilization more than $10 \%$ higher than the random protocol. In this scenario, the utilization is reduced for the random protocol since there is no coordination for transmissions among neighbor nanonodes. 


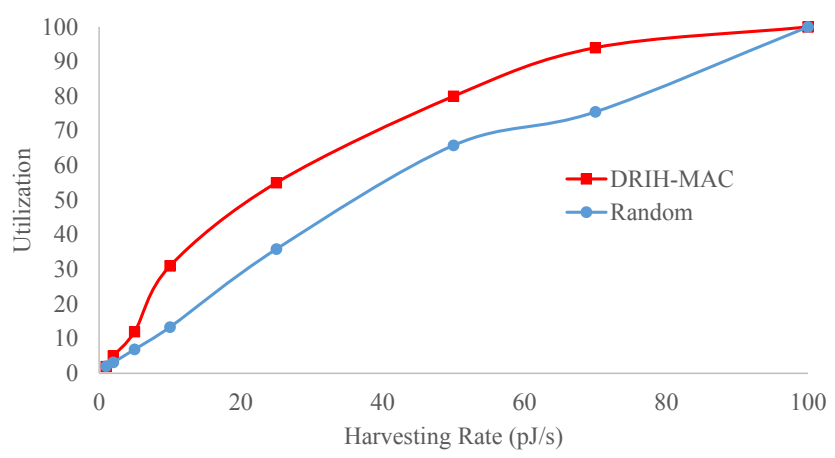

Fig. 15: Energy Utilization of a Single Link in a Network

Figure 16 illustrates the energy utilization for various numbers of nanonodes. With an increase in the density of the network, more collisions among simultaneous transmission occurs due to the existence of hidden terminals. Consequently, the energy utilization drops with the increase in the number of nanonodes. However, DRIH-MAC still outperforms the Random protocol. One interesting observation is that the DRIH-MAC protocol performance for the energy harvesting rate of $10 \mathrm{pJ} / \mathrm{s}$ is very close to the performance of the Random protocol with a higher energy harvesting rate, i.e., $25 \mathrm{pJ} / \mathrm{s}$. This observation represents the efficiency of DRIH-MAC in energy utilization.

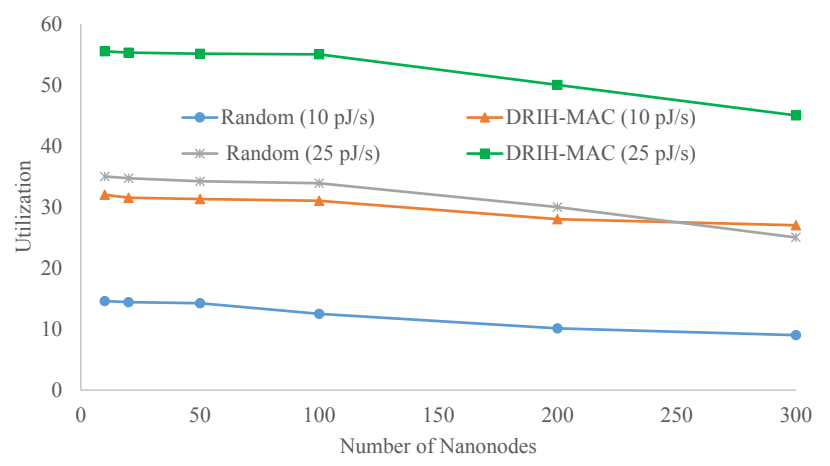

Fig. 16: Energy Utilization versus Number of Nanonodes

\section{DRIH-MAC in Medical Applications}

In this section, we evaluate the performance of a basic application with the protocols and schemes we have developed so far. We evaluate the performance of a medical monitoring application in which nanonodes transfer their measurement of intra and/or on body phenomena to the micro and macro domains through a nanocontroller. This application is representative of a large category of nanonode applications. We evaluate the deployment of DRIH-MAC for a particular application, but DRIH-MAC is a general MAC protocol that could be used in many applications with similar energy models and application requirements, as long as the proper design selection of system parameters are met in terms of number of nanonodes, energy storage capacity, and energy harvesting rate. For example, we believe our techniques could be used in IoT, environment monitoring, and plant monitoring applications. Here, we show this design for a particular blood monitoring application that can help the diagnosis, prevention, and cure of many diseases such as diabetes, blood pressure disorders, and various infections.

The scenario is as follows. The nanonodes are distributed in the veins along the arm. The number of nanonodes required for effective measurement depends on the fabrication of devices and required measurement accuracy. However, with artery diameters between $0.5-10 \mathrm{~mm}$ and nanonodes of $10 \mu \mathrm{m}$, there could be 110 nanonodes at each point, with the assumption that nanonodes do not occupy more than $0.5-1 \%$ of artery diameter, to avoid interference with blood flow. We assume a network of 300 nanonodes uniformly distributed in an area of $30 \times 10 \times 10$ $\mathrm{cm}^{3}$. Moreover, we consider nanonodes to be operating in an aqueous environment since between $50-70 \%$ of human body is composed of water. Refer to Appendix $B$ for the derivations of path loss in an aqueous environment.

The nanonodes sense various blood components, such as glucose or cholesterol. There is one nanocontroller that will gather results and send them to the interface outside of the body. The nanocontroller has higher energy storage, $300 \mathrm{pJ}$, and can harvest energy at the maximum harvest rate of 20 $\mathrm{pJ} / \mathrm{s}$. It is assumed that the nanonodes will harvest energy from the motion of the body. Various parts of the body can generate vibrations over a wide range of rates, e.g., from $1 \mathrm{~Hz}$ (person tapping his foot) to more than $300 \mathrm{~Hz}$ (person running) [27], [28]. Considering the nanowire energy harvester model [4], these vibration rates will result in energy harvesting rates of approximately 0.2 to $10 \mathrm{pJ} / \mathrm{s}$. The other parameters of our scenario are presented in Table IV]

TABLE IV: Simulation Parameters

\begin{tabular}{|l|l|}
\hline Duration & $60 \mathrm{~s}$ \\
\hline Packet Generation Interval & $1 \mathrm{~s}$ \\
\hline RTR Packet Size & $25 \mathrm{~B}$ \\
\hline DATA Packet Size & $250 \mathrm{~B}$ \\
\hline Harvesting Rate & $0.2-5 \mathrm{pJ} / \mathrm{s}$ \\
\hline Nanonode Communication Range & $15 \mathrm{~cm}$ \\
\hline Forwarding Value $(\mathrm{g})$ & 1 \\
\hline
\end{tabular}

Not all nanonodes can communicate directly with the nanocontroller. Therefore, they are responsible for forwarding the traffic of other nanonodes towards the nanocontroller. The main metric to evaluate the performance of DRIH-MAC is to show the delay in receiving recent blood monitoring information. We are assuming that this information is required at least every 5 seconds. Figure 17 illustrates the delay corresponding to various energy harvesting rates. As can be seen, for any energy harvesting rate larger than the $0.5 \mathrm{pJ} / \mathrm{s}$, the delay would be less than 5 seconds for DRIH-MAC while the energy harvesting must be $1 \mathrm{pJ} / \mathrm{s}$ for random MAC to achieve delay less than 5 seconds. Notice again this delay is mainly due to the waiting time to harvest enough energy for communication.

Increasing the number of nanonodes would reduce the delay in delivering the blood monitoring information as illustrated in Figure 18. This reduction is due to the fewer number of hops that a packet would travel to reach the destination.

\section{RELATED WORK}

There exists a large body of literature on MAC protocol design for sensor networks [29] and pulse-based Ultra-WideBand (UWB) networks [30]. However, these MAC protocols cannot directly be used in nanonetworks because they do not consider either the limitations of nanodevices (i.e., limited processing 


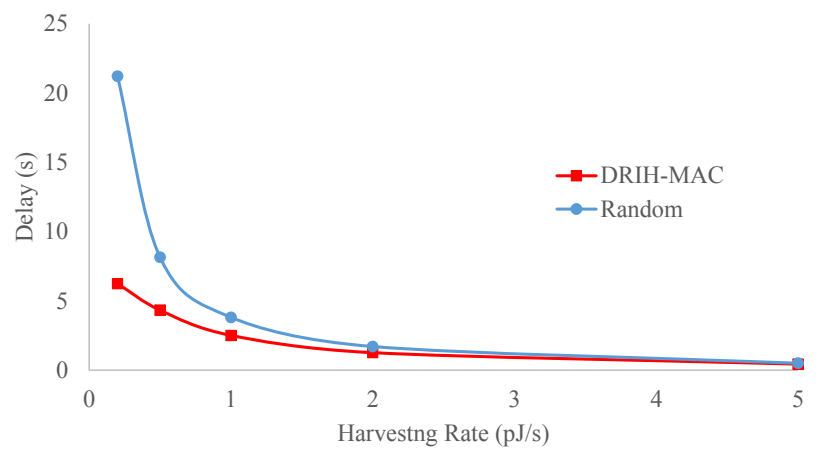

Fig. 17: Delay for Various Harvesting Rates

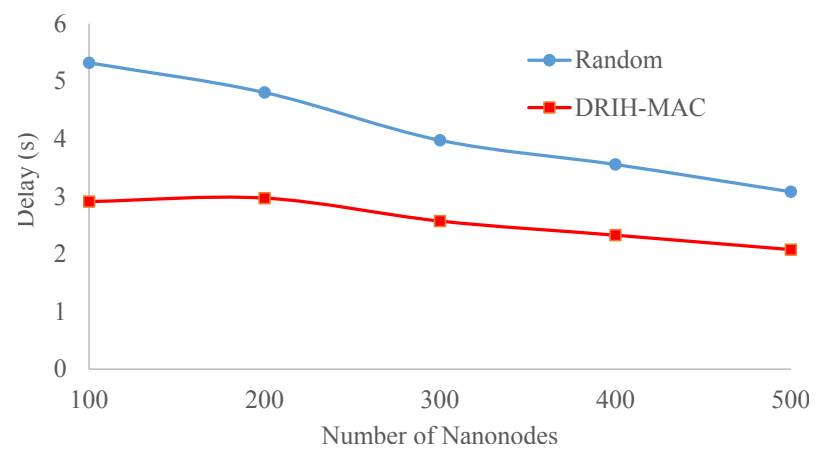

Fig. 18: Delay for Various Number of Nanonodes Harvesting Rate $=1 \mathrm{pJ} / \mathrm{s}$

capacity, limited memory, limited energy storage), energy harvesting, or the characteristics of the Terahertz band. The main limitation of nanodevices results from the limited energy that can be stored in nano-batteries or nano-capacitors. Therefore, energy harvesting-aware protocols are required. Recently, energy harvesting-aware designs for sensor networks have been studied. However, most of the studies cannot be applied to nanonetworks. First, the energy storage of nanonodes is limited while in the previous works it is mainly considered infinite or extremely large. Second, most of the schemes (e.g., [6], [7]) are too complex to run on nanonodes. Finally, the energy harvesting rate is usually considered very close the consumption rate in previous work. However, in nanonetworks, the harvesting rate for most energy sources is likely to be smaller than the energy consumption rate. Moreover, the majority of the existing MAC protocols for wireless networks have been designed for bandlimited channels. In nanonetworks, the $\mathrm{THz}$ channel provides nanodevices with an almost $10 \mathrm{THz}$ wide window. Furthermore, carrier-sensing techniques in classic MAC protocols cannot be used in pulse-based communication systems since there is no carrier for sensing. Only some solutions [30] proposed for Impulse Radio Ultra Wide Band (IR-UWB) networks could be considered, but their complexity limits their usefulness in the nanonetworks. Moreover, the characteristics of the $\mathrm{THz}$ band as well as the limited processing capabilities of nanodevices are the major factors that necessitate the redesign of protocols for nanonetworks.

Receiver-initiated protocols have been investigated in duty cycle sensor networks [31], [32]. However, those methods cannot be used directly for energy harvesting environments due to the stochastic properties of energy harvesting. Moreover, it is not clear how much these receiver-initiated protocols can be effective in energy harvesting-aware protocols. In our work proposed in this paper, we investigated the use of receiverinitiated protocols for energy-harvesting nanonetworks.

Protocol design for nanonetworks is still in its early stages. In our previous work [26], we introduced an optimization model to find the optimal values for packet size, code weight, and repetition. We designed a multiobjective function problem to address several functions such as energy consumption, end to end delay, and communication reliability. In [12], the authors proposed PHLAME, which mainly focuses on applying the optimal values of code weight and repetition for packet transmission to address energy consumption and reliability. The performance of the proposed protocol is analytically studied in terms of energy consumption, delay, and achievable throughput by using models of the $\mathrm{THz}$ channel. However, the implementation feasibility and energy efficiency evaluation of the method are still open questions. Later, an energy harvesting-aware and light-weight MAC protocol was proposed in [13]. This protocol attempts to achieve fair throughput and optimal channel access among nanosensors that are controlled by a nanocontroller, representing a centralized topology. Towards this end, the critical packet transmission ratio is defined, which is the maximum allowable ratio between the transmission time and the energy harvesting time. A nanosensor has to harvest more energy than it consumes to reach perpetual data transmission. Our proposed protocol, DRIH-MAC, in contrast with previous MAC protocols for nanonetworks, is a receiver-initiated protocol that can adapt itself to various energy harvesting rates.

\section{CONCLUSIONS}

In this paper, we described a receiver-initiated MAC protocol for electromagnetic wireless nanonetworks. Nanonodes in such a network rely on energy harvesting to supply energy for their communication. Our distributed receiver-initiated protocol, DRIH-MAC, takes into account the energy harvesting properties of nanonodes that form an ad hoc network. DRIH-MAC is scalable with the increase in the number of nanonodes and also leads to a low number of collisions. This protocol is adaptable to be deployed in a large variety of nanonetwork applications, where delay and packet loss are not hard requirements for quality of service. We evaluated the performance of DRIH-MAC and compared it against a random MAC protocol in the context of a medical monitoring application. Simulation results showed that DRIH-MAC utilized energy better.

In the future, we will investigate the use of DRIH-MAC in other applications such as Internet of Nano-Things [33] or a network of nano-robots [34]. Both the traffic model and application requirements are different in these nanonetwork applications. A potential solution could be a hybrid design of centralized and distributed topologies to address the needs of such networks. Moreover, nodes in these nanonetworks would have more mobility. Therefore, extending DRIH-MAC to support these scenarios is another future research direction.

\section{APPENDIX A}

\section{CECS FUNCTIONALITY}

Here, we analyze and prove how CECS ensures packet exchange between a receiver and transmitter although they have not scheduled communication during one particular time slot. 
The proof follows two steps. We first show that there exists a probability for being in any of the levels for a policy, and then show that the probability is non-zero for two nanonodes using the patterns resulting in packet exchanges.

\section{A. Energy Harvesting and Consumption Processes}

The energy harvesting and consumption processes can be modeled as a Markov chain, which we denote as $M$. For each nanonode, energy harvesting follows a random variable, while energy consumption is defined based on a set of available actions on how much energy is to be consumed. We consider a discrete time model, in which the time is slotted into intervals of unit length. In timeslots of a cycle, some energy is harvested and added to the energy storage, and similarly some energy is consumed and deducted from the energy storage based on the consumption scheme. We assume that the energy storage is ideal and there is no significant leakage.

We denote the system states by $S=S_{1}, S_{2}, \cdots, S_{s}$, where $s=C+1$ for energy storage of capacity $C \cdot E_{\min }$ units of energy, $E_{\min }$ denotes the unit of energy, e.g., 1 pJ.

It is assumed that there are always packets ready for transmission. Being in the transmitter and receiver roles, will consume $E_{t x}$ and $E_{r x}$ units of energy, respectively, to exchange a DATA packet and a RTR packet. The consumption strategy of a nanonode, i.e., the number of times being the transmitter and receiver per cycle, is denoted as $a_{(i, j)}$, for $i, j \geq 0$, which is selected from

$A=\left\{a_{(0,0)}, a_{(1,0)}, a_{(0,1)}, a_{(1,1)}, \cdots, a_{(m, n)}, a_{(m+1, n)}, a_{(m, n+1)}, a_{(m)}\right.$

The action $a_{(i, j)}$ corresponds to being the receiver for $i$ times and the transmitter of $j$ times in the cycle, where the sum of the energy consumption, denoted as $E_{k}$, cannot exceed the maximum consumption per cycle, $E_{c}, 0 \leq E_{c} \leq C$, i.e., $i \cdot E_{r x}+j \cdot E_{t x} \leq E_{c} \quad \forall i, j$. We denote $S_{A}$ as the number of members of $A$. For simplicity of presentation, we define $i \cdot E_{r x}+j \cdot E_{t x}$ as $E_{k}$, with the corresponding $a_{k}, 1 \leq k \leq S_{A}$. The consumption action taken for each state of energy depends on the design of consumption model. For example, in an optimum design, there would be only one action per state.

The probabilities of transferring between states depend on the current state, the amount of energy harvested, and the action taken. Formally, the state transitions can be written as

$$
P\left(S_{i}, S_{j}\right)=p_{u}, \quad \sum_{x=1}^{s} P\left(S_{i}, S_{x}\right)=1,1 \leq i, j \leq s,
$$

and $j$ is specified as

$$
j=i+h_{u}-E_{k},
$$

where $E_{k}<i, i+h_{u} \leq s, 0 \leq u \leq D$, and $1 \leq k \leq S_{A}$. The value of $j$ represents the energy state after the harvesting of $h_{u}$ units and consumption of $E_{k}$ units of energy for action $a_{k}$ taken. The condition $E_{k}<i$ limits the actions which can be taken to avoid consuming more energy than is harvested and stored. The condition $i+h_{u} \leq s$ limits the harvested energy to the available capacity of energy storage. When $j=1$, the system falls into the out of energy state, i.e, the node has consumed all of energy that it has stored and harvested. When $j=s$, the system falls into the full energy state, i.e., even after consumption, the system has stored and harvested up to the capacity $C$.
Now, we show that this Markov chain for the energy harvesting and consumption is ergodic, which means it would have a stationary solution.

\section{B. Markov Chain Properties and Relation with CECS}

Lemma A.1: The Markov chain $M$ is irreducible.

Proof: From Equation (2), it is straightforward to show there any other state can be accessed in one or many transitions, i.e., $P^{n}\left(S_{i}, S_{j}\right)>0$.

Lemma A.2: $P\left(S_{0}, S_{0}\right)>0$

Proof: When no energy exists and harvested, the system stays in the same state, i.e., $P\left(S_{0}, S_{0}\right)>0$

Lemma A.3: The Markov chain is ergodic

Proof: Using Lemmas (A.1) and A.2, it is concluded that $M$ is ergodic.

Corollary A.4: For any ergodic Markov chain, there is a unique stationary solution with probabilities $\pi_{i}>0,1 \leq i \leq s$.

Theorem A.5: The probability of two neighbor nanonodes being in the 1 of their CECS scheduling pattern simultaneously is non-zero.

Proof: Consider two arbitrary nanonodes are not in their first state at same time and they are in states $i$ and $j$ respectively, with their corresponding patterns denoted as $r_{i}$ and $r_{j}$. The rotation of patterns means that the probability of being in any rotation offset of a pattern would be

$$
p_{r}=\frac{1}{l}>0,
$$

where $l$ is the number of neighbors for a nanonode. We define this probability for nanonodes $i$ and $j$ as $p_{r_{i}}$ and $p_{r_{j}}$, respectively. Let us define $V$ as the event that two nanonodes are in $1 \mathrm{~s}$ of their CECS scheduling pattern simultaneously. Next, we can write

$$
\operatorname{Pr}(V)=\pi_{i} \cdot p_{r_{i}} \cdot \pi_{j} \cdot p_{r_{j}}>0
$$

If two nanonodes are in their first states at the same time, then the probability that they will not stay there in the future would be

$$
1-\pi_{1} \cdot \pi_{1}>0
$$

because $\pi_{1} \neq 1$. Therefore, they will go to two other states and then Equation (4) will apply to them.

\section{APPENDIX B}

\section{Calculation of Path Loss in Aqueous Environment}

Here, we calculate the path loss in an aqueous environment. Path loss consists of two main components: absorption loss and free space propagation loss. We present the calculation of these losses in the 0.1-10 THz in an aqueous environment.

For liquids, transmittance is related to absorbance A (not to be confused with absorptance) as

$$
A=-\log (T)=-\log \left(\frac{I}{I_{0}}\right),
$$

where $I$ is the intensity of radiation (after transmission through liquid) and $I_{0}$ is the intensity of radiation before it passes through the material.

Similarly, the transmission (transmissivity) is given by

$$
T=\left(\frac{I}{I_{0}}\right)=10^{-\alpha \cdot d}
$$


where $d$ represents the distance and $\alpha$ is the attenuation coefficient.

From (6) and (7), it can be written $A=\alpha \cdot d \cdot \log (10)$. The value of $\alpha$ is $\alpha=\frac{4 \cdot \pi \cdot k}{\lambda_{0}} \cdot d$, where $\lambda_{0}$ is the vacuum wavelength (the wavelength of the light in free space), and $k$ is the imaginary part of the refractive index. The refractive index of materials varies with the wavelength. In opaque media, the refractive index is a complex number, where the real part describes refraction, and the imaginary part accounts for absorption.

The attenuation coefficient $(\alpha)$ can be approximated with the absorption coefficient. We used the values of the absorption coefficient, collected by Segelstein [35], [36], to calculate the absorption loss in $\mathrm{dB}$. We plot the absorption loss in Figure 19 for $0.1-1 \mathrm{THz}$ and distances up to $5 \mathrm{~cm}$.

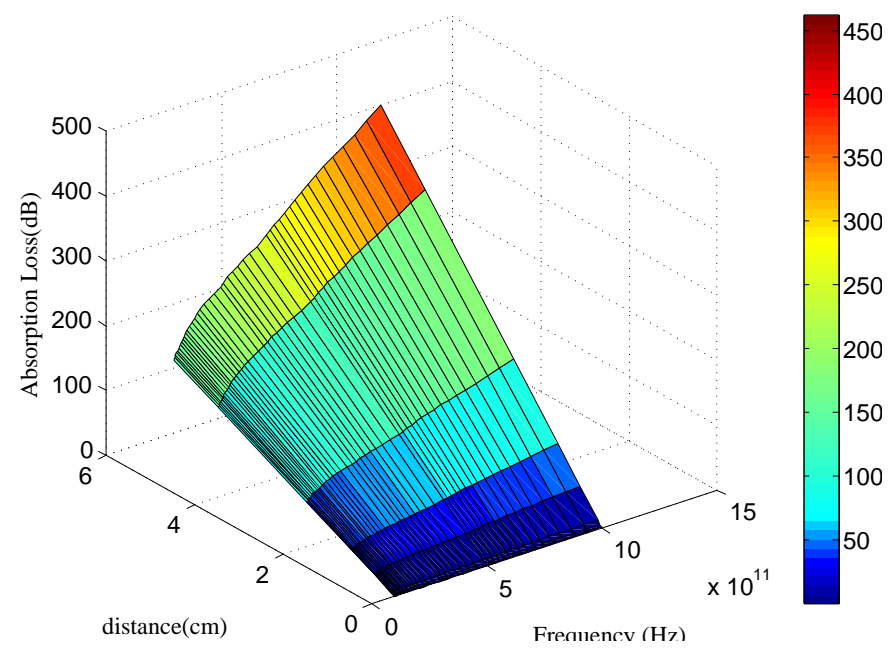

Fig. 19: Absorption Loss at Different Distances for Water

As shown, the absorption loss is less than $100 \mathrm{~dB}$ in less than $1 \mathrm{~cm}$. Also, for lower frequencies, e.g., 100-300 $\mathrm{GHz}$, the absorption loss would be under $10 \mathrm{~dB}$. Note that fat and muscles have lower attenuation values [37]. Therefore, the calculations here are valid for inside the body communication.

Free-space propagation loss is another parameter that affect the effective range of communication. The free-space propagation loss is defined as

$$
P_{L}(f, d)=\left(\frac{4 \pi f d}{c}\right)^{2},
$$

where $f$ is the frequency of interest, $d$ is the distance between the transmitter and the receiver, and $c$ is the speed-of-light in a vacuum. The free space propagation loss in $\mathrm{dB}$ can be represented as

$$
P_{L}=10 \cdot \log \left(P_{L}(f, d)\right) .
$$

Figure 19 illustrates the path loss with the change of frequency in the range of $0.1-1 \mathrm{THz}$ for $0.01,0.1$ and $1 \mathrm{~m}$ distances.

Combining the absorption loss and propagation loss shows that communication in water would only be possible in centimeter distances. Figure 21 represents the total loss (sum of absorption and free-space loss) for various distances at $100 \mathrm{GHz}$ and $300 \mathrm{GHz}$. As can be viewed the loss would be less than $100 \mathrm{~dB}$ at distance less than $0.5 \mathrm{~m}$.

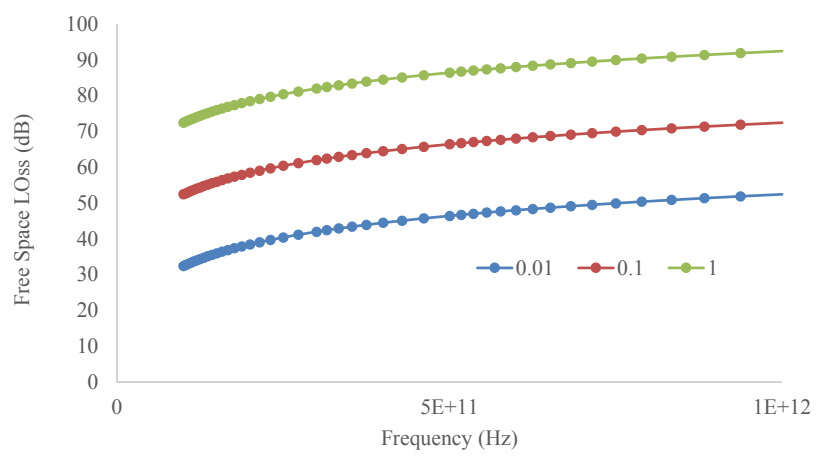

Fig. 20: Propagation Loss at Different Distances

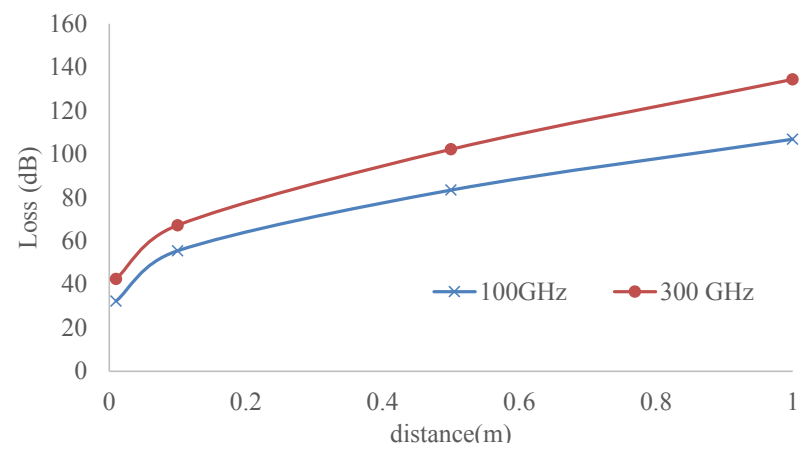

Fig. 21: Path Loss at Different Distances

\section{ACKNOWLEDGMENT}

The authors sincerely thank the anonymous reviewers for insightful comments and constructive suggestions which helped improve the technical quality of the paper significantly. The work of S. K. Das was partially supported by NSF grants under award numbers CNS-1404677, IIS-1404673, and CNS-1355505.

\section{REFERENCES}

[1] S. Mohrehkesh and M. C. Weigle, "RIH-MAC: Receiver-initiated harvesting-aware MAC for nanonetworks," in Proc. of the First ACM Annual International Conference on Nanoscale Computing and Communication (NANOCOM), 2014, pp. 6:1-6:9.

[2] I. F. Akyildiz, F. Brunetti, and C. Blazquez, "Nanonetworks: A new communication paradigm," Computer Networks, vol. 52, no. 12, pp. 2260-2279, 2008.

[3] I. F. Akyildiz and J. M. Jornet, "Electromagnetic wireless nanosensor networks," Nano Comm. Networks, vol. 1, no. 1, pp. 3-19, 2010.

[4] J. Jornet and I. Akyildiz, "Joint energy harvesting and communication analysis for perpetual wireless nanosensor networks in the terahertz band,' IEEE Trans. Nanotechnol., vol. 11, no. 3, pp. 570-580, May 2012.

[5] S. Xu, B. J. Hansen, and Z. L. Wang, "Piezoelectric-nanowire-enabled power source for driving wireless microelectronics," Nat Commun, vol. 1, Oct. 2010.

[6] R.-S. Liu, K.-W. Fan, Z. Zheng, and P. Sinha, "Perpetual and fair data collection for environmental energy harvesting sensor networks," IEEE/ACM Trans. on Networking, vol. 19, no. 4, pp. 947 -960, Aug. 2011.

[7] V. Sharma, U. Mukherji, V. Joseph, and S. Gupta, "Optimal energy management policies for energy harvesting sensor nodes," IEEE Trans. on Wireless Commun., vol. 9, no. 4, pp. 1326-1336, April 2010. 
[8] K. Tutuncuoglu and A. Yener, "Optimal power policy for energy harvesting transmitters with inefficient energy storage," in 46th Annиal Conference on Information Sciences and Systems (CISS), March 2012, pp. $1-6$.

[9] S. Mohrehkesh and M. C. Weigle, "Optimizing energy consumption in terahertz band nanonetworks," to appear in IEEE JSAC: Molecular, Biological, and Multi-Scale Communications Series, December 2014, available at: http://www.cs.odu.edu/ smohrehk/pub/JSAC2014.pdf.

[10] J. Jornet and I. Akyildiz, "Channel modeling and capacity analysis for electromagnetic wireless nanonetworks in the terahertz band," IEEE Transactions on Wireless Communications, vol. 10, no. 10, pp. 32113221, October 2011.

[11] _ - "Low-weight channel coding for interference mitigation in electromagnetic nanonetworks in the terahertz band," in IEEE International Conference on Comm. (ICC), Jun 2011, pp. 1 -6.

[12] J. M. Jornet, J. C. Pujol, and J. S. Pareta, "PHLAME: A physical layer aware MAC protocol for electromagnetic nanonetworks in the terahertz band," Nano Communication Networks, vol. 3, no. 1, pp. 74 - 81, 2012.

[13] P. Wang, J. M. Jornet, M. A. Malik, N. Akkari, and I. F. Akyildiz, "Energy and spectrum-aware MAC protocol for perpetual wireless nanosensor networks in the terahertz band," Ad Hoc Networks, vol. 11, no. 8, pp. $2541-2555,2013$

[14] G. D. S. Boisseau and B. A. Seddik, Electrostatic Conversion for Vibration Energy Harvesting. InTech, 2012.

[15] J. Jornet and I. Akyildiz, "Channel capacity of electromagnetic nanonetworks in the terahertz band," in IEEE International Conference on Communications (ICC), May 2010, pp. 1 -6.

[16] J. M. Jornet and I. F. Akyildiz, "Information capacity of pulse-based wireless nanosensor networks," in Proc. of IEEE SECON, 2011, pp. 8088

[17] R. Cid-Fuentes, J. Jornet, I. Akyildiz, and E. Alarcon, "A receiver architecture for pulse-based electromagnetic nanonetworks in the terahertz band," in IEEE International Conference on Communications (ICC), June 2012, pp. 4937-4942.

[18] S.-Y. Ni, Y.-C. Tseng, Y.-S. Chen, and J.-P. Sheu, "The broadcast storm problem in a mobile ad hoc network," in Proc. of ACM MobiCom, 1999, pp. 151-162.

[19] N. Wisitpongphan, O. Tonguz, J. Parikh, P. Mudalige, F. Bai, and V. Sadekar, "Broadcast storm mitigation techniques in vehicular ad hoc networks," IEEE Wireless Comm., vol. 14, no. 6, pp. 84-94, Dec. 2007.

[20] T. Otsuji, S. Tombet, A. Satou, M. Ryzhii, and V. Ryzhii, "Terahertzwave generation using graphene: Toward new types of terahertz lasers," IEEE Journal of Selected Topics in Quantum Electronics, vol. 19, no. 1, 2013.

[21] R. Merris, Graph Theory. John Wiley \& Sons, Inc., 2000.

[22] D. A. Grable and A. Panconesi, "Nearly optimal distributed edge colouring in $\mathrm{O}(\log \log \mathrm{n})$ rounds," in Proc. of the Eighth Annual ACM-SIAM Symposium on Discrete Algorithms, Philadelphia, PA, USA, 1997, pp. $278-285$.

[23] S. Gandham, M. Dawande, and R. Prakash, "Link scheduling in wireless sensor networks: Distributed edge-coloring revisited," Journal of Parallel and Distributed Computing, vol. 68, no. 8, pp. 1122 - 1134, 2008.

[24] M. Gorlatova, A. Wallwater, and G. Zussman, "Networking low-power energy harvesting devices: Measurements and algorithms," IEEE Trans. on Mobile Computing, vol. 12, no. 9, pp. 1853-1865, 2012.

[25] G. Piro, L. A. Grieco, G. Boggia, and P. Camarda, "Nano-sim: simulating electromagnetic-based nanonetworks in the network simulator 3," in Proc. of Workshop on NS- 3, Cannes, France, Mar. 2013.

[26] S. Mohrehkesh and M. C. Weigle, "Optimizing communication energy consumption in perpetual wireless nanosensor networks," in Proc. of the IEEE Globecom, Atlanta, GA, December 2013, pp. 545-550.

[27] S. Roundy, "On the effectiveness of vibration-based energy harvesting," Journal of Intelligent Material Systems and Structures, vol. 16, no. 10, pp. 809-823, 2005.

[28] S. Roundy, P. K. Wright, and J. Rabaey, "A study of low level vibrations as a power source for wireless sensor nodes," Computer Communications, vol. 26, no. 11, pp. 1131 - 1144, 2003.

[29] I. Akyildiz, W. Su, Y. Sankarasubramaniam, and E. Cayirci, "A survey on sensor networks," IEEE Comm. Magazine, vol. 40, no. 8, pp. 102 114, Aug. 2002.

[30] A. Gupta and P. Mohapatra, "A survey on ultra wide band medium access control schemes," Comput. Netw., vol. 51, no. 11, pp. 2976-2993, Aug. 2007.

[31] Y. Sun, O. Gurewitz, and D. B. Johnson, "RI-MAC: A receiver-initiated asynchronous duty cycle MAC protocol for dynamic traffic loads in wireless sensor networks," in Proc. of the 6th ACM SenSys, 2008, pp. $1-14$.

[32] L. Tang, Y. Sun, O. Gurewitz, and D. Johnson, "PW-MAC: An energyefficient predictive-wakeup MAC protocol for wireless sensor networks," in Proc. of IEEE INFOCOM, 2011, pp. 1305-1313.

[33] I. Akyildiz and J. Jornet, "The internet of nano-things," IEEE Wireless Communications, vol. 17, no. 6, pp. 58 -63, December 2010.

[34] D. D. Nicolas Boillot and J. Bourgeois, "Using nano-wireless communications in micro-robots applications," in Proc. of The First ACM Annual International Conference on Nanoscale Computing and Communication (NANOCOM), 2014, pp. 10:1-10:9.

[35] D. Segelstein, "The complex refractive index of water," Master's thesis, University of Missouri, Kansas City, Missouri, USA, 1981.

[36] J. Xu, K. Plaxco, S. Allen, J. Bjarnason, and E. Brown, "0.15 THz absorption of aqueous salts and saline solutions," Applied Physics Letters, vol. 90, no. 3, pp. 1908-19011, Jan 2007.

[37] D. Werber, A. Schwentner, and E. M. Biebl, "Investigation of RF transmission properties of human tissues," Advances in Radio Science, vol. 4, pp. 357-360, 2006.

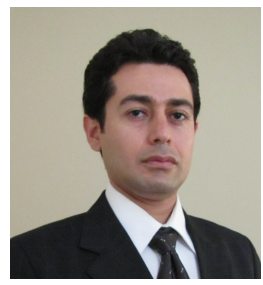

Shahram Mohrehkesh (S'13) Shahram Mohrehkesh received the $\mathrm{PhD}$ degree of Computer Science from Old Dominion University, Norfolk, VA in 2015. His research interests include nanonetworks, big data analysis, wireless networks, mobile computing, and green computing and communication. His current research is about energy harvesting-aware solutions for nanonetworks. He has produced over 20 publications in journals and conferences related to wireless and mobile networks. He has received 2 nd rank at the Nokia Mobile Data Challenge at 2012, the best paper award in ACM MiseNet 2013 and 2014. He was selected as the award recipient of Phi Kappa Phi awards from the college of Science at ODU in April 2015.

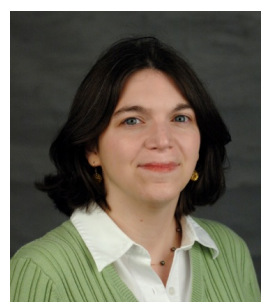

Michele C. Weigle (M'07) received her Ph.D. from the University of North Carolina at Chapel Hill in 2003. She is an Associate Professor of Computer Science at Old Dominion University. Her research interests include wireless and mobile networks, network simulation and modeling, information visualization, and web science. She and her collaborators have received over \$2 million in funding from the National Science Foundation for research in wireless networks, sensor networks, and Internet traffic modeling and simulation. She has produced over 35 publications in journals and conferences related to wireless networking. She is also the author of three book chapters and the co-editor of one of the first books on vehicular networks, Vehicular Networks: From Theory to Practice, published by CRC Press in 2009. Dr. Weigle has participated on the technical program committees (TPC) for several international conferences and serves on the steering committee for the International Workshop on Intelligent Vehicular Networks and as TPC Vice-Chair for the IEEE Workshop on Networking for Intelligent Vehicles and Infrastructures (NiVi) and the IEEE Vehicular Networks and Applications Workshop (Vehi-Mobi). Dr. Weigle is a member of ACM, ACM SIGCOMM, ACM-W, IEEE, and IEEE ComSoc. 


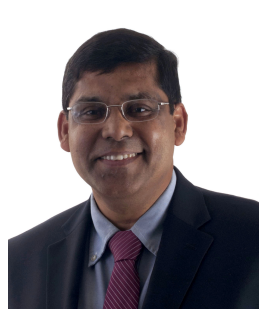

Sajal K. Das (F'15) is the Chair of the Computer Science Department and Daniel St. Clair Endowed Chair at Missouri University of Science and Technology, Rolla. During 1999-2013 he was a University Distinguished Scholar Professor of Computer Science and Engineering, and founding director of Center for Research in Wireless Mobility and Networking (CReWMan) at the University of Texas at Arlington. During 2008-2011, Dr. Das was a Program Director at the National Science Foundation in the Computer Networks and Systems Division. His current research interests include wireless sensor networks, smart environments (smart healthcare and smart energy management), cyber-physical systems, mobile and pervasive computing, security and privacy, biological and social networks.

$\mathrm{He}$ has published more than 250 journal articles, more than 400 peerreviewed conference papers, and 51 book chapters. He coauthored four books Smart Environments: Technology, Protocols, and Applications (Wiley 2005); Handbook on Securing Cyber-Physical Critical Infrastructure: Foundations and Challenges (Morgan Kaufman 2012); Mobile Agents in Distributed Computing and Networking (Wiley 2012); and Principles of Cyber-Physical Systems (Cambridge University Press, 2015).

Dr. Das is a recipient of the IEEE Computer Societys Technical Achievement Award for pioneering contributions to sensor networks and mobile computing, IEEE Region-5 Outstanding Engineering Educator Award; and 10 Best Paper Awards.

$\mathrm{He}$ is the Editor-in-Chief of Elseviers Pervasive and Mobile Computing journal, and Associate Editor of several journals including IEEE Transactions on Mobile Computing, ACM Transactions on Sensor Networks. A co-founder of the IEEE WoWMoM, IEEE PerCom and ICDCN conferences, he has also served as General and Program Chair of numerous IEEE and ACM conferences. He received his B.S. degree from Calcutta University, M.S. degree from Indian Institute of Science, Bangalore and Ph.D. degree from University of Central Florida, all in Computer Science. Dr. Das is a fellow of the IEEE. 